\title{
NASA seeks a better, faster distribution of space data
}

\begin{abstract}
Washington. The US National Aeronautics and Space Administration (NASA), faced with political pressure to increase its links with the wider community, is urging scientists who fly experiments on its spacecraft to share their data with the public and other researchers more quickly than in the past.

The space agency is also considering privatizing - and perhaps breaking up - its vast archive of data from past space missions. Many scientists say that they support such an idea, provided the service is not scaled back to save money.
\end{abstract}

Daniel Goldin, the head of NASA, has told space scientists that he thinks the practice of allowing individual researchers exclusive access to taxpayer-funded data should be curtailed or stopped.

Outside scientists working on NASAsponsored missions, as well as researchers granted time on NASA instruments such as the Hubble Space Telescope, traditionally have a proprietary period during which only they can use the data. This period is typically between six and 18 months, after which the data are archived and made available to the entire research community.

But the recent trend on projects such as the Pentagon-funded Clementine Moonmapping mission, or the observing campaign for last year's comet impact with Jupiter, has been to release the data immediately. Keen to 'reinvent' its culture, this has now become NASA's preferred model; for example, the agency has asked science teams for the 1997 Cassini Saturn mission to reduce the proprietary data period to as little as nine months.

Stamatios Krimigis of the Johns Hopkins University's Applied Physics Laboratory, a principal investigator on the Cassini mission, supports the space agency's push toward quicker dissemination, saying that in the past some researchers have "sat on their data" for far too long.

Another Cassini team member, Jonathan Lunine of the University of Arizona, agrees that "we should err in the direction of making [the data] available as soon as possible". But, he warns, NASA should also give team scientists enough money and resources to sort out bugs in the data and to format the data for general distribution. Otherwise the released data could be of poor quality.

Defenders of a proprietary period argue that external scientists who spend years developing a flight experiment should have first crack at publishing the results. If the period is too short, they say, team members can spend a considerable amount of time preparing the data for everyone else, and thus lose their competitive advantage.

A new NASA 'science policy guide' released this week, which covers a wide range of issues affecting the NASA science community, acknowledges that the question of data rights involves "sometimes contradictory factors", from the question of intellectual property rights to the risk of publishing inaccurate results.

The guide, which was written by France Cordova, NASA's chief scientist, and the agency's science administrators, says that data policy for each NASA research project will in future be established on a "case-bycase basis".

Meanwhile, the space agency is looking for ways to hand over the management of its archive of data from past spacecraft missions to a non-government entity - or entities. Much of the data is now kept at the National Space Science Data Center (NSSDC) at the Goddard Space Flight Center in Maryland, which provides tens of thousands of researchers each year with free data over the Internet, or with tapes and CD-ROMs sold at minimal cost.

Last week, NASA called for ideas on how the NSSDC could be privatized. Few believe a privately run service would cost the government less; as there is no real "market" beyond the research community, true commercialization is probably not an option. But a private operator may offer a better and more efficient service than the NSSDC. Many scientists say the current system, although it has improved over recent years, is outmoded and difficult to use.

The data archive might be broken up by discipline, with one centre handling highenergy astrophysics data, for example, and another handling planetary geology data. The Space Telescope Science Institute in Baltimore, Maryland, which archives data from the Hubble telescope, could be a model for these kinds of distributed centres.

The advantage of this approach, says Krimigis, is that the data would be treated with "understanding and tender loving care" by researchers who work with them every day, and who can best format them for the rest of the science community.

However these data issues are settled, the new NASA science policy guide - which is due to be made available on the Internet at http://www.nasa.gov, under "Hot Topics" indicates that NASA-funded scientists are no longer expected to serve just the science community, but also the national economy, politicians, and school children, among others.

Under a section called the "Social Contract", the document states that "both the public and the political system expect benefits broader than purely scientific ones to be derived from NASA research programmes and missions". Tony Reichhardt

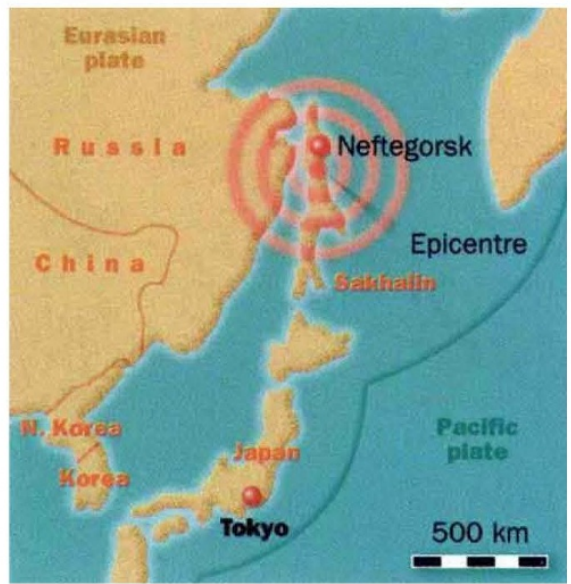

Rough encounter: the Sakhalin earthquake occured in region where plates meet

\section{Limited knowledge in Russian quake zone}

London. Poorly designed buildings, relatively few seismic stations and an earlier reluctance to acknowledge eastern Russia as an active earthquake zone each appear to have contributed to the 2,000 fatalities in last month's magnitude 7.1 earthquake on the island of Sakhalin.

Russia's Interfax news agency reported last week that the local prosecutor's office has instituted criminal proceedings for infringing building safety codes after 19 fivestorey buildings, constructed during the 1960 s in the city of Neftegorsk collapsed.

Russian seismologists, however, are divided over why conventional structures had been built in an area well known for its seismicity (Sakhalin has experienced dozens of earthquakes since records began, including a magnitude 7.1 earthquake in 1971).

Some claim the Soviet authorities leant on geologists to underplay the earthquake risk in the region - despite the fact that such phenomena occur regularly - to save money on otherwise expensive buildings.

But others disagree, claiming the problem was mere ignorance. "We really did not know much about this area during the Khrushchev building era of the 1960s," says one former Russian seismologist now working in the West. "The north of Sakhalin was relatively inaccessible, and our instruments were very inaccurate," he says, adding that surveys carried out at ten-year intervals gradually changed the Russian estimation of the zone's seismicity. "But for a long time seismic zoning for the whole of Siberia was very primitive."

Meanwhile, 60 of Russia's 180 seismic stations - including one at Okha, the town nearest Neftegorsk - have been closed as a result of budget cuts over the past year, according to Oleg Starovoyt, director of the Russian Academy of Sciences' Geophysical Service. Many were in dangerous seismic regions, he says, adding that stations still operating are badly underfinanced. E.M. 